\title{
CARBONIZATION OF THE DIII-D TOKAMAK
}

\author{
by \\ G.L. JACKSON, J. WINTERS,* S. LIPPMANN, \\ T.W. PETRIE, J.C. DeBOO, J.R. FERRON, \\ D.N. HILL, ${ }^{\dagger}$ D.P. SCHISSEL, T.S. TAYLOR
}

This is a preprint of a paper to be presented at the 9th International Conference on Plasma Surface Interactions, Bournemouth, United Kingdom, May 21-25, 1990, and to be printed in the Proceedings.

*IPP, KFA, Jülich, FRG, Association EURATOM-KFA

tLawrence Livermore National Laboratory

Work supported by

Department of Energy

Contract DE-AC03-89ER51114
GENERAL A'TOMICS PROJECT 3466
DECEMBER 1990

GENERAL ATOMICS 


\title{
Carbonization of the DIII-D Tokamak
}

\author{
G.L. Jackson, J. Winter, ${ }^{*}$ S. Lippmann,
}

T.W. Petrie, J.C. DeBoo, J.R. Ferron, D.N. Hill, ${ }^{\dagger}$ D.P. Schissel, T.S. Taylor

General Atomics Inc., P.O. Box 85608, San Diego, CA 92138, USA

\begin{abstract}
The DIII-D tokamak has been carbonized by the application of a carbon film covering all plasma facing surfaces. Carbonization was done in order to reduce the metal impurity influx and central metal accumulation especially during beam heated $\mathrm{D}^{\circ} \rightarrow \mathrm{D}^{+} \mathrm{H}$-mode discharges. After carbonization, nickel impurity line radiation was reduced by a factor of 10 during the ohmic phase of the discharge and up to a factor of 30 during the $\mathrm{H}$-mode phase. The reduction of metal impurities also produced a reduction of total radiated power and allowed high current operation. After carbonization, the highest plasma current in a double null divertor, and the highest stored energy ever achieved on DIII-D were observed, $3 \mathrm{MA}$ and 3.6 MJ respectively. A toroidal beta, $\beta_{\mathrm{T}}=5.1 \%$, at full field, $2.1 \mathrm{~T}$, was obtained. On the first day after carbonization, $\mathrm{H}$-mode density profiles were more peaked than pre-carbonization discharges.
\end{abstract}

\section{Introduction}

In recent years, substantial progress has been achieved in fusion research with better control of plasma wall interactions. Specifically, the introduction of low Z materials such as graphite and carbon films has allowed operation in regimes of higher plas na temperatures and stored energy, larger effective neutron yields, and higher $\beta_{\mathrm{T}}[1-3]$. The DIII-D tokamak uses graphite on $40 \%$ of the plasma facing surface

* Institute of Plasmaphysics, KFA, Jülich, Federal Republic of Germany, Association EURATOMKFA.

$\dagger$ Lawrence Liverrrore National Laboratory. 
including all surfaces exposed to higher than average heat flux. This coverage has been adequate for much of the DIII-D experimental program but at plasma currents at or above $\sim 2 \mathrm{MA}$ metal accumulation, which consists primarily of nickel, the major constituent of the DIII-D Inconel 625 vacuum vessel, can seriously degrade beam heated $\mathrm{D}^{\circ} \rightarrow \mathrm{D}^{+} \mathrm{H}$-mode discharges. The metal influx and, particularly, metal accumulation in the central region of the plasma can lead to a large fraction of radiated power, $\mathrm{P}_{\mathrm{rad}} / \mathrm{P}_{\text {in }}<1$. A high metal influx can produce locked modes and a high fraction of radiated power can cause a loss of confinement due to radiation collapse.

In order to reduce metal influx in DIII-D, a carbon film was deposited on all plasma facing surfaces. A glow discharge consisting of helium and methane was used to deposit this carbon film in a manner similar to TEXTOR [4]. We will present the details of carbonization and the subsequent effects on tokamak discharges in this paper.

\section{Description of Carbonization}

Carbonization was first done in December 1989 and the procedures are similar to those performed on TEXTOR $[4,5]$. With the exception of the third carbonization at room temperature, all carbonization sessions were accompanied by a pre- and post- bake of the vessel to $350^{\circ} \mathrm{C}$. After all sessions, helium glow wall conditioning (HeGWC) was performed for 20 to $30 \mathrm{~min}$ to further desorb remaining hydrogenic species in the surface and near surface layers of the carbon film before commencing tokamak operations. A short (3 to $7 \mathrm{~min}$ ) HeGWC session is also routinely performed before every tokamak discharge [3].

The DIII-D glow discharge system consists of two anodes separated $180^{\circ}$ toroidally. A total glow current of seven amperes corresponds to an average wall current of $8 \mu \mathrm{A} \mathrm{cm}^{-2}$. The details of this glow system are described elsewhere [3,6]. A surface deposition probe at the radius of the outside midplane Inconel tiles and $75^{\circ} \mathrm{C}$ toroidally from the closest glow anode was used to monitor the film thickness [7]. The average 
film thickness was also calculated from (a) gas throughput measurements and (b) total methane consumption. Residual gas analysis was used to determine the fraction of methane deposited during the glow discharge. The difference in film thickness determined by these three measurements was $30 \%$ or less. Both pure methane and a mixture of methane and helium were used during these carbonizations. A methane/helium mixture more readily desorbs excess hydrogen during the carbonization process and hence can reduce particle fueling on subsequent tokamak discharges [5]. However a carbonization session with a pure methane glow discharge followed by a post-carbonization bake showed no deleterious effects, such as high carbon or $\mathrm{D}_{2}$ particle influxes.

\section{Effect of Carbonization on Tokamak Discharges}

In order to characterize the effects of carbonization, a series of similar beam heated discharges $\left(I_{p}=2 \mathrm{MA}\right.$, single null divertor, $\mathrm{D}^{\circ} \rightarrow \mathrm{D}^{+}, \mathrm{B}_{\mathrm{T}}=2.1 \mathrm{~T}$ ) were done immediately before and after carbonization. We will first discuss these discharges in Sections 3a-3c and then present results from high current discharges, long term trends after carbonization and other post-carbonization effects.

\section{3a. Impurity reduction after carbonization}

Both the fraction of radiated power and impurity line radiation, measured with a UV spectrometer, was reduced after carbonization, as shown in Fig. 1. Nickel line radiation was reduced by a factor of $\sim 10$ during the ohmic and L-mode phases of the discharges. Edge nickel impurity lines, i.e. those lines such as NiXVII which predominantly radiate in the edge plasma region, also decreased by an order of magnitude when compared with pre-carbonization discharges. OVI radiation was reduced by a factor of $\sim 6$ although as discussed in Section $3 \mathrm{~d}$, the decrease in oxygen levels did not last for as many discharges as did the reduction in metal impurities. Metal accumulation in the center of the discharge, inferred from NiXXV and NiXXVI was 


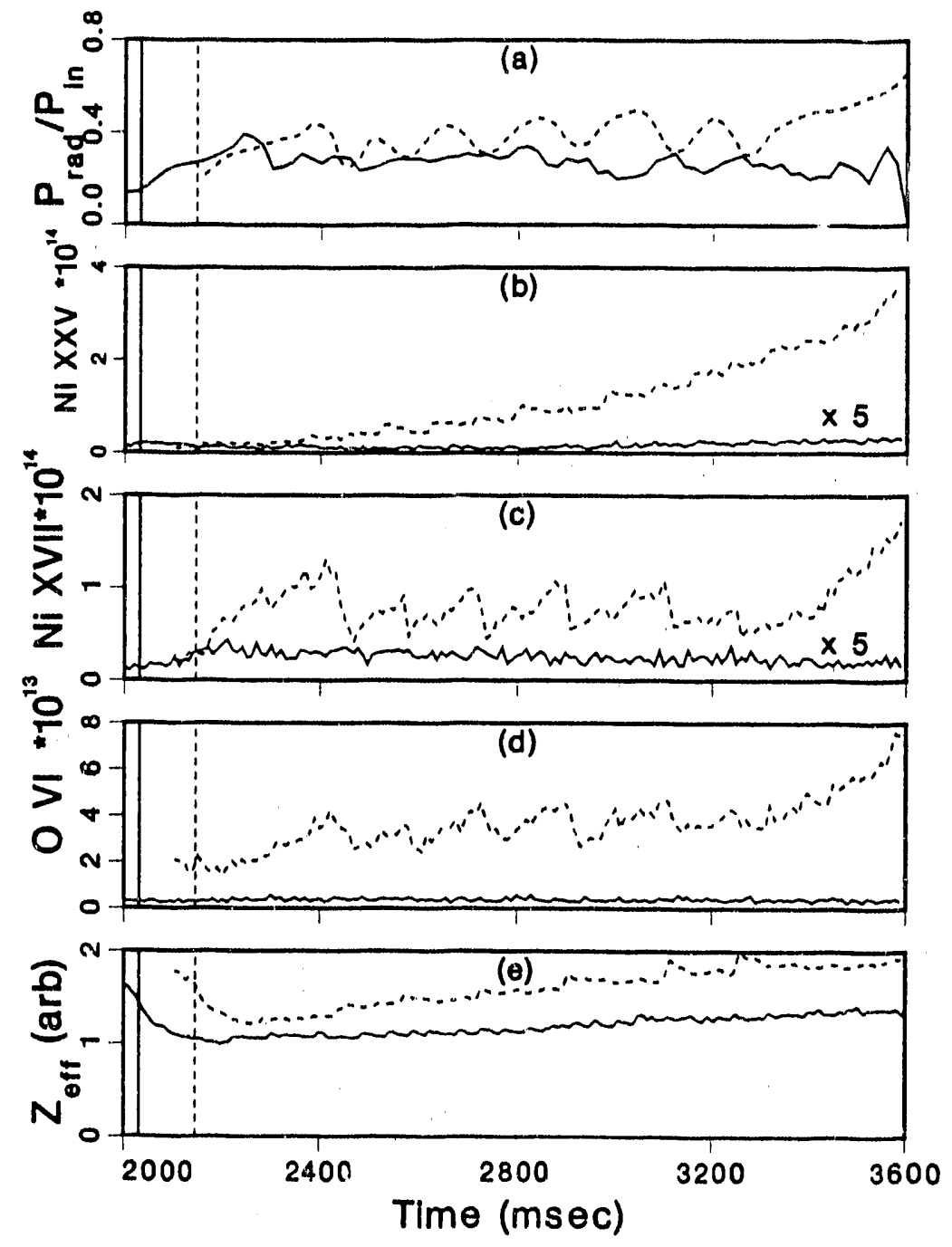

Fig. 1. Nickel and oxygen impurity line radiation are reduced by a factor of 5 to 30 after carbonization (solid lines, \#67901) when compared with a similar discharge before carbonization (dashed lines, \#67822); 2.0 MA single null divertor (SND), $2.1 \mathrm{~T}, 6.5 \mathrm{MW}$ $D^{\circ} \rightarrow D^{+}$. The fraction of radiated power, $P_{\text {rad }} / P_{\text {in }}$ and $Z_{\text {eff }}$ are also reduced. Only the beam heated portion is displayed and the $L$ to $\mathrm{H}$ transition time is shown as a vertical line for each discharge. 
substantially lower, up to a factor of $\sim 30$ during $\mathrm{H}$-mode, which is consistent with the less peaked radiated power profiles measured by the bolometer array and plotted in Fig. 2.

Although radiated power decreased after carbonization, power flow to the divertor region, inferred from an absolutely calibrated infra-red (I-R) camera, increased by more than a factor of two. The time between ELMs and the average power loss due to ELMs, $\mathrm{P}_{\mathrm{ELM}}$, increased. For example, a comparison of two similar discharges $\left(\mathrm{I}_{\mathrm{P}}=2.0 \mathrm{MA}, \beta_{t}=2.1 \mathrm{~T}, \mathrm{D}^{\circ} \rightarrow \mathrm{D}^{+}\right)$during $\mathrm{H}$-mode showed that before carbonization $\mathrm{P}_{\mathrm{rad}}, \mathrm{P}_{\mathrm{elm}}$, and $\mathrm{P}_{\mathrm{div}}$ were $0.95 \mathrm{MW}, 3.2 \mathrm{MW}$ and $0.64 \mathrm{MW}$ respectively $\left(\mathrm{P}_{\text {beam }}+\mathrm{P}_{\text {ohmic }}=5.6 \mathrm{MW}\right)$. After carbonization $\mathrm{P}_{\mathrm{rad}}, \mathrm{P}_{\mathrm{elm}}$, and $\mathrm{P}_{\mathrm{div}}$ were $1.8 \mathrm{MW}$, 1.5 MW, and 0.87 MW respectively $\left(P_{\text {beam }}+P_{\text {ohmic }}=5.2 \mathrm{MW}\right)$.

\section{3b. Peaked density profiles during $\mathrm{H}$-mode}

Discharg's immediately after carbonization are accompanied by a reduction of $\mathrm{D}_{\alpha}$ line radiation in the divertor region, shown in Fig. 3, and also at all other locations around the machine viewed by the $\mathrm{D}_{\alpha}$ photodiode array. Gas fueling was also reduced for these discharges with lower $D_{\alpha}$ emission.

Post-carbonization discharges with lower $\mathrm{D}_{\alpha}$ recycling also exhibited a peaking of the electron density profile as shown in Fig. 4(a). The total kinetic pressure profile is plotted in Fig. 4(c). Kinetic pressure was calculated using profile measurements froru Thomson scattering $\left(\mathrm{n}_{e}\right.$ and $\left.\mathrm{T}_{e}\right), \mathrm{CO}_{2}$ interferometer $\left(\mathrm{n}_{e}\right)$, charge exchange recombination $\left(T_{i}\right)$, and $Z_{\text {eff }}\left(n_{i}\right)$. The kinetic stored energy in this analysis is within $20 \%$ of the stored energy calculated from magnetic equilibrium analysis [8]. For comparison, a discharge with a flat density profile which is typical of most DIII-D high current $\mathrm{H}$-mode discharges is also shown in Fig. 4. Although this 1.8 MA discharge occurred on the second day after carbonization, it is typical of 2 MA pre-carbonization discharges. It is presented here because a complete set of diagnostic measurements was 

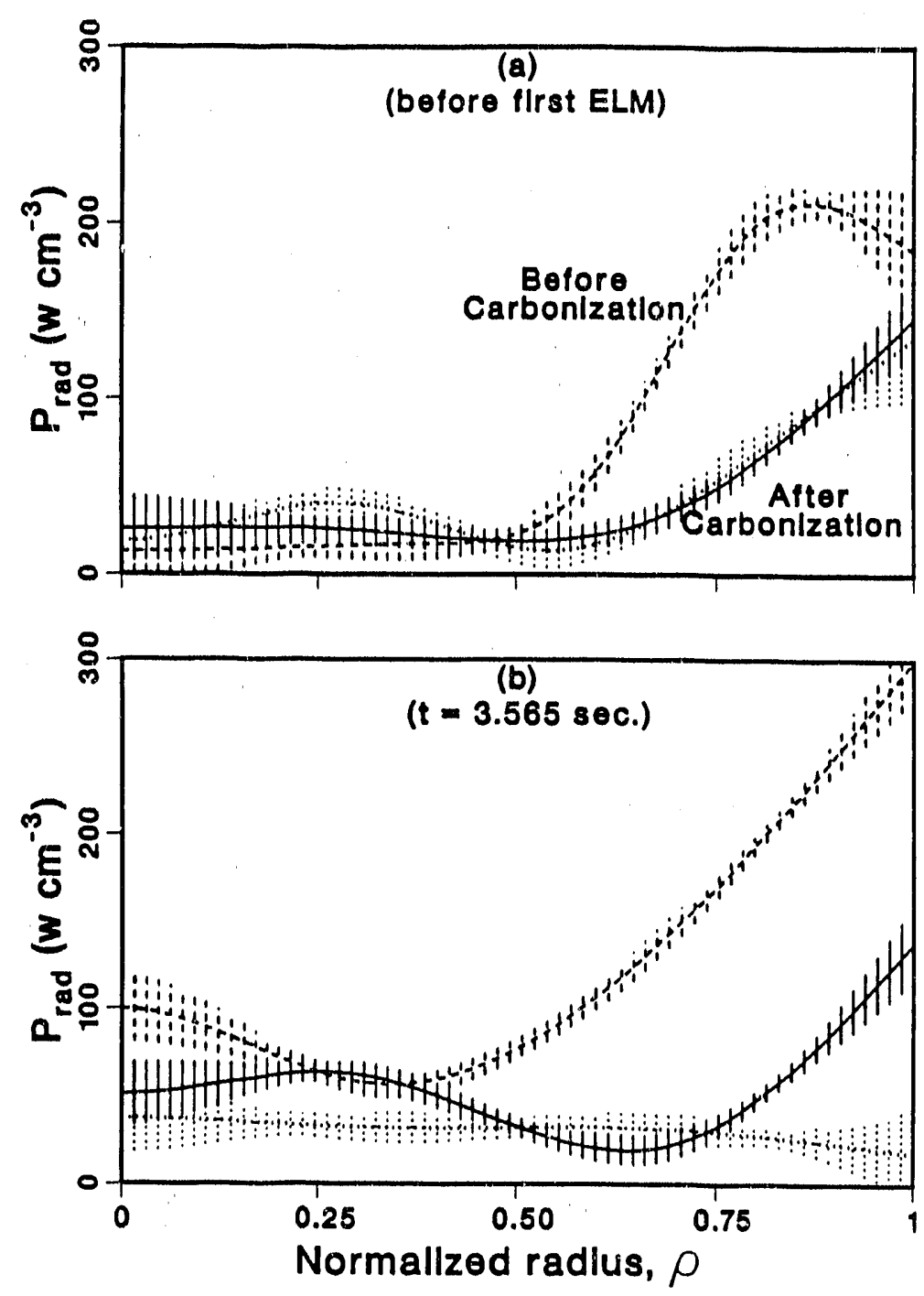

Fig. 2. Radiated power profiles inferred from the bolometer array as a function of the normalized flux for similar discharges before carbonization (dashed lines, \#67822), the first day after carbonization (dotted lines, \#67875), and the second session after carbonization (solid lines, \#67901). Discharge conditions are the same as Fig. 1 and profiles are shown during $\mathrm{H}$-mode before the first ELM (a) and at $3.565 \mathrm{sec}$ in the steady state portion of the $\mathrm{H}$-mode discharge (b). 

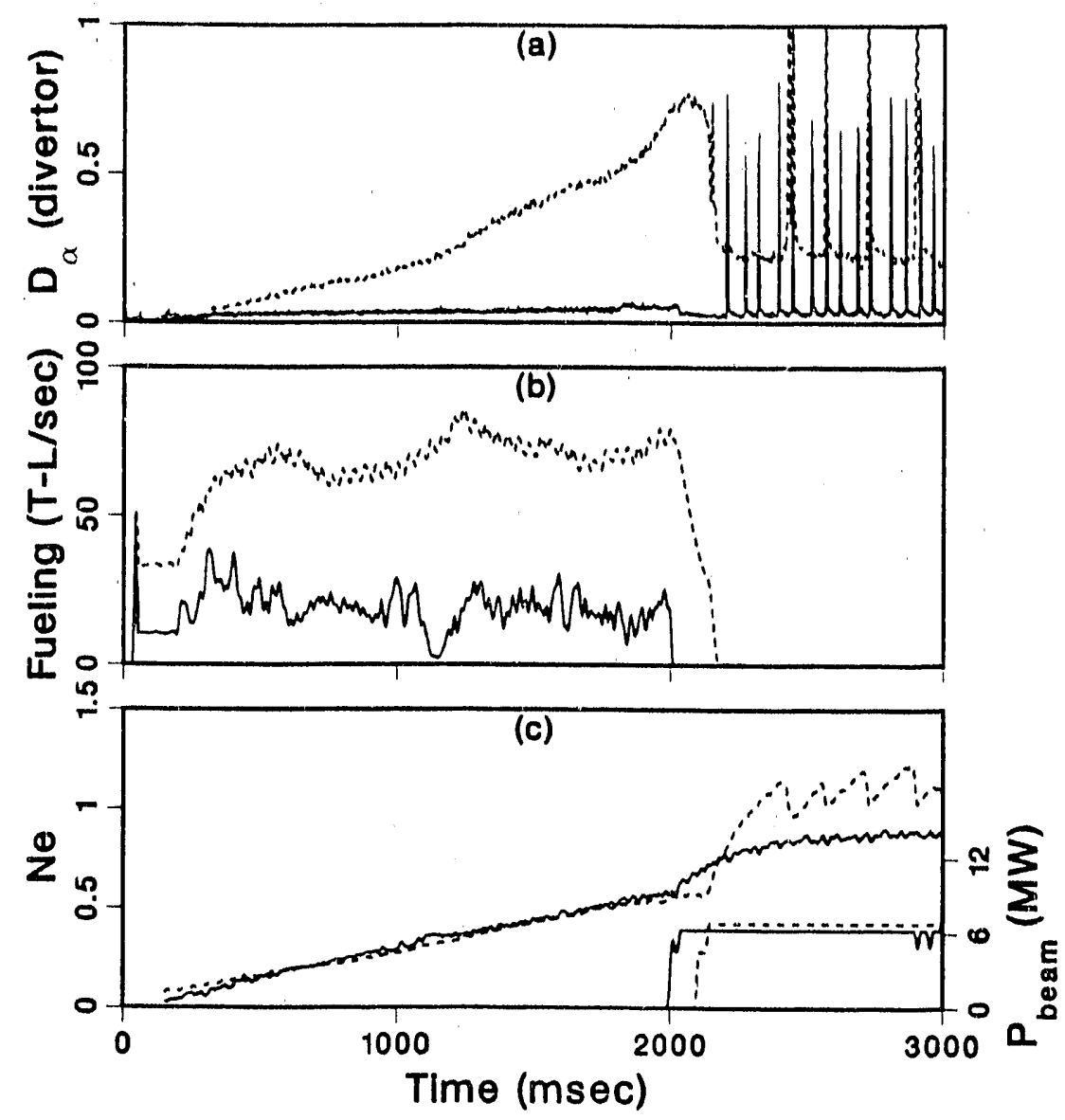

Fig. 3. $D_{\alpha}$ emission from the divertor region and $\Delta t$ ELM decreases while gas fueling increases for a discharge on the first day after carbonization (solid lines) when compared to a pre-carbonization discharge. Discharge conditions are the same as in Fig. 1.

not available for the pre-carbonization characterization series of discharges, precluding a full kinetic analysis of these discharges. The density peaking factor is defined as

$$
\mathrm{R}_{n_{\ell}}=\frac{n_{\ell}(0)}{\iiint n_{\ell}(r, \theta) d v}
$$

where $\ell$ represents either ions, $R_{n i}$ or electrons, $R_{n e}$. 

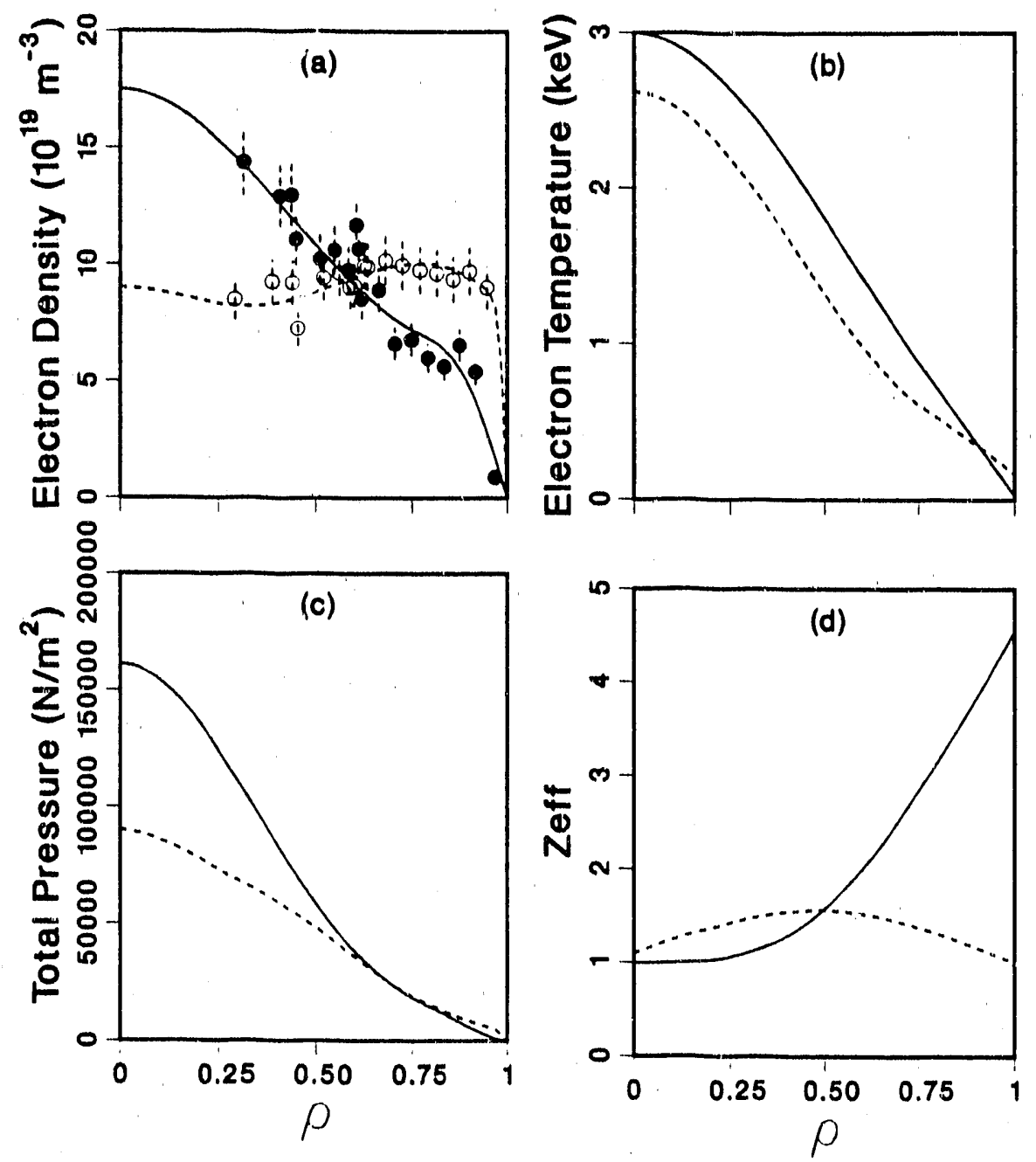

Fig. 4. Electron density and total kinetic pressure profiles are more peaked for discharges on the first day after carbonization (solid lines, $2 \mathrm{MA}$, $2.1 \mathrm{~T}, \mathrm{D}^{\circ} \rightarrow \mathrm{D}^{+} \mathrm{SND}$ ) than either subsequent tokamak discharges (dashed lines, 1.8 MA, 2.1 T, $\mathrm{D}^{\circ} \rightarrow \mathrm{D}^{+}$, SND) or similar precarbonization discharges. $Z_{\text {eff }}$ on axis is near unity.

$\mathrm{R}_{n e}$ is $\sim 1.0$ and $\sim 2.0$ for the peaked and flat discharges in Fig. 4 respectively. Profiles from these discharges were both analyzed near the time of peak stored energy in between ELMs. Central $Z_{\text {eff }}$ also decreased on discharges where peaked profiles were observed, shown in Fig. 4(d). Ion density profiles can be inferred from the electron density and $Z_{\text {eff }}$ profiles. The ion density peaking factor was higher for the post-carbonization discharge in Fig. 4 with lower recycling. 


\section{3c. Confinement scaling and high current operation after carbonization}

$\mathrm{H}$-mode global energy confinement in $2 \mathrm{MA}$ beam heated discharges was similar before and after carbonization at input powers above $5 \mathrm{MW}$, as plotted in Fig. 5, even though the time between ELMs changed by more than a factor of 2 . The confinement time in Fig. 5 is at the time of peak stored energy which is measured by magnetic probe and psi loop arrays [8]. There is less energy loss per ELM after carbonization, as shown in Fig. 5(c), but power flow to the divertor region is higher for post-carbonization discharges with lower recycling and lower $\overline{\mathrm{n}}_{\mathbf{e}}$. Energy confinement in double null discharges was also similar before and after carbonization. For both pre- and post-carbonization, stored energy was an offset linear function of $P_{i n}$ and confinement at constant power scaled as $\tau_{E} \sim 0.84 \mathrm{I}_{\mathrm{p}}^{0.3 \alpha_{4} \pm 0.2}[9]$. This is within the error bars of the previously observed scaling, $\tau_{E} \sim \mathrm{I}_{\mathrm{p}}^{1.0}$, from a more extensive data set.

The primary motivation to carbonize DIII-D was to reduce metai influx and allow higher current operation. After the 2 MA carbonization characterization discharge 3 were completed plasma current was increased to $3 \mathrm{MA}$ in a double null divertor configuration. During this rur period, 79 discharges with $\mathrm{I}_{\mathrm{p}} \geq 2.5 \mathrm{MA}$ were accomplished as part of the experimental program. Previously only one 2.5 MA full length diverted discharge had been successfully completed in DIII-D. The highest stored energy ever achieved in DIII-D was observed, 3.6 MJ, and a 2.8 MA discharge at full field, $2.1 \mathrm{~T}$, was obtained with a reactor relevant volume averaged toroidal beta of $5.1 \%[11]$.

\section{3d. Long term changes in impurity influx after carbonization}

An important issue for routine operation with carbonization is the number of discharges before the carbon film is eroded and impurity radiation increases to its previous level. The behavior of OVI and NiXVII are plotted in Fig. 6 over a span of 


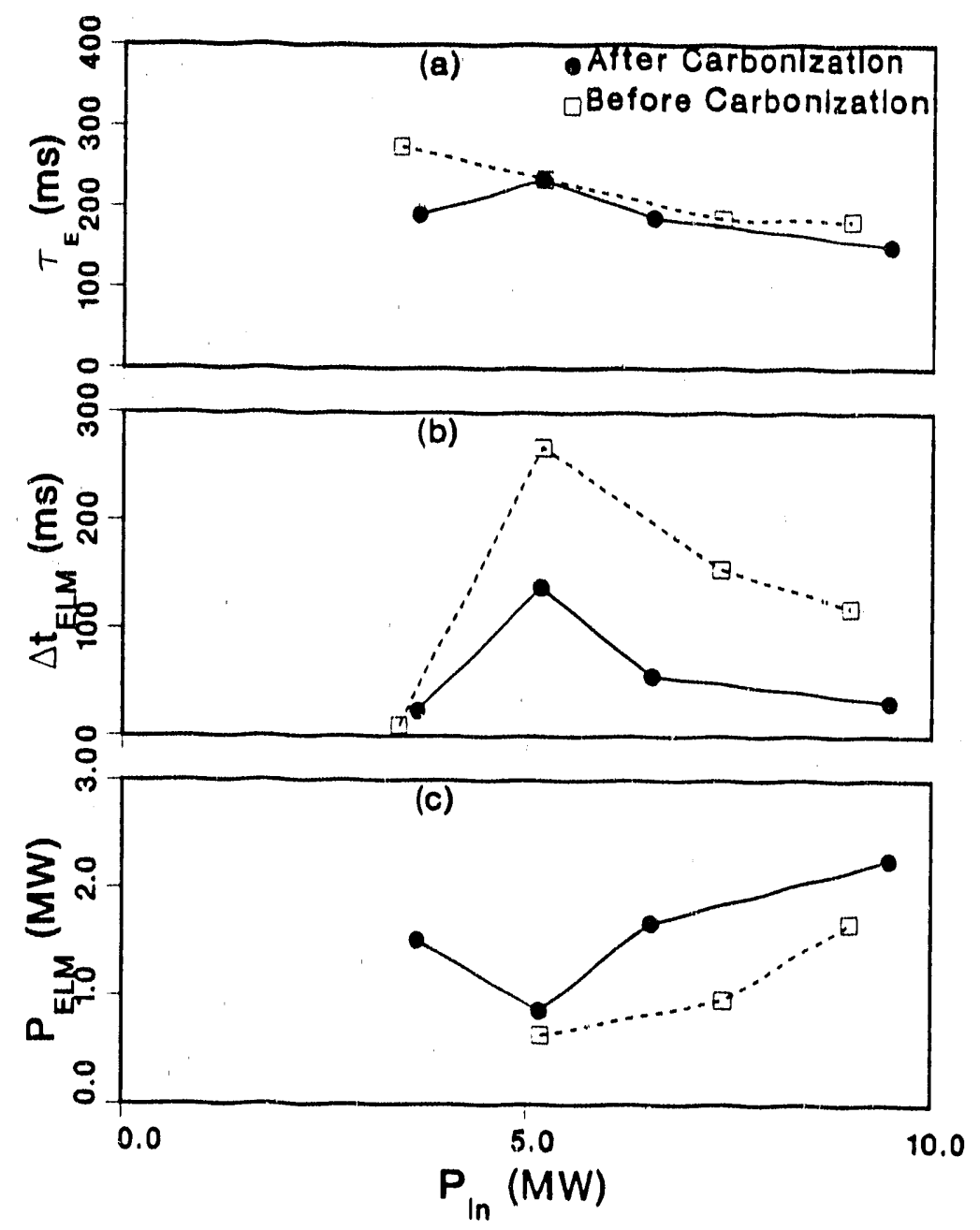

Fig. 5. Energy confinement time is similar before (dashed line) and afier carbonization except near the $L$ to $H$ transition power threshold. For input powers above $5 \mathrm{MW}\left(\mathrm{P}_{\mathrm{in}}=\mathrm{P}_{\text {ohmic }}+\mathrm{P}_{\text {beam }}\right)$ the time between ELMs is reduced by a factor of 2 or more while the power loss due to ELMs, defined by Eqn (2), increases.

$\sim 300$ plasma discharges and 3 sessions of carbonization. The line intensities in Fig. 6 were measured at $1.0 \mathrm{sec}$ during the ohmic current ramp portion of the discharge to minimize changes in discharge conditions such as auxiliary power and peak plasma current. The rate of increase in OVI line intensity is higher after each successive carbonization. For example, after the second carbonization in Fig. 6, the OVI intensity was initial.y at $13 \%$ of its pre-carbonization average and returned to $86 \%$ after 130 
discharges. The influx of nickel, begins to increase dramatically it $\sim 70$ discharges after the second carbonization during a period when plasma currents 2.5 MA were routinely obtained with neutral beam injection power up to $18 \mathrm{MW}$.

\section{3e. Other post-carbonization effects}

Ion Bernstein Wave (IBW) heating experiments have also observed lower impurity influx after carbonization. Before carbonization, metal influx during IBW injection could often irigger locked modes. This impurity influx was primarily nickel, from the DIII-D Inconel walls, but titanium was also observed (the IBW Faraday shield is coated with $\mathrm{TiC}$ ). Nickel line radiation increased dramaticall-" during IBW injection. For example, NiXVIII increased by a factor of $\sim 3$ for an injected power of $0.5 \mathrm{MW}$ before carbonization. After carbonization the increase in NiXVIII was less than $30 \%$. The increase in radiated power during IBW injection, $\triangle \mathrm{P}_{\mathrm{rad}}$, was also reduced after carbonization. A comparison of similar discharges before and after caibonization indicates $\Delta \mathrm{P}_{\mathrm{red}} / \mathrm{P}_{\mathrm{rf}}=0.66$ and 0.37 respectively. IBW injected power was increased to $1.0 \mathrm{MW}$ in post-carbonization discharges and was limited by power supply constraints on that day ( $\mathrm{P}_{\mathbb{B B W}}^{\mathrm{Max}} \sim 2 \mathrm{MW}$ ) and not plasma wall interactions.

\section{Discussion}

There are two potential disadvant:ıges in carbonizing a tokamak such as DIII-D: (1) neutral beam drift duct heating caused by $D_{2}$ desorption from the carbnn film interacting with the fast beam particles and (2) excessive $\mathrm{D}_{2}$ fueling and carbon influx from the deuterium rich carbon film. However, neither of the potential problems significantly affected DIII-D operation.

$D_{\alpha}$ radiation in the neutral beam drift ducts exhibitcd an $\sim 5$ discharge decay constant as neutral beam injection was initiated after carbonization. As shown in Fig. 3, the external gas fueling, $D_{\alpha}$ line radiation and $\bar{n}_{e}$ were all reduced on the first day after carbonization. 


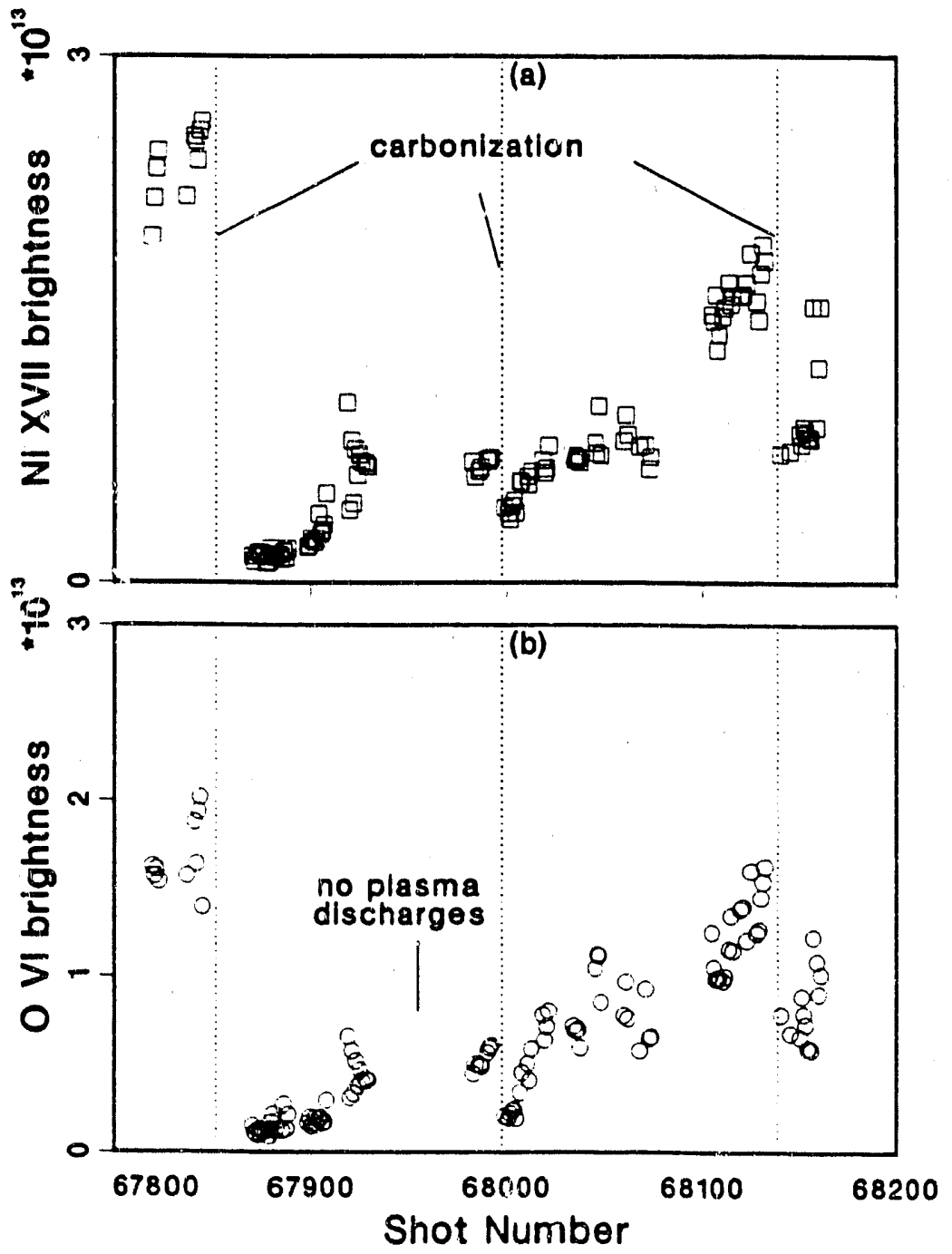

Fig. 6. Long term trends in OVI and NiXVII emission at $1.0 \mathrm{sec}$ in the ohmic current ramp of tokamak discharges during the first carbonization campaign which included three carbonization sessions. During a period of high current plasma discharges (up to $3 \mathrm{MA}$ ) after the second carbonization, a sharp increase in NiXVII emission was observed. 
For similar discharges during the pre- and post-carbonization characterization there was no appreciable increase in carbon influx. However an increase in carbon influx was observed during IBW injection after the third carbonization shown in Fig. 6 at room temperature. Although this carbonization successfully suppressed metal impurities and allowed high current operation during a subsequent session of tokamak discharges, the carbon influx during IBW injection produced an incremental increase in radiated power which was higher than in pre-carbonization IBW experiments. As discussed previously, the first two carbonization sessions were successful in reducing radiated power during IBW injection discharges.

Peaking of the density and pressure profile as observed in DIII-D H-mode post-carbonization discharges is important for the next generation of fusion devices. A peaked density profile and a high central kinetic pressure concentrates a larger fraction of particles in the high temperature portion of the discharge where the probability for fusion reactions is highest, and thus suck a configuration is more efficient and perhaps essential for a viable reactor design. Thus it is important to understand why this peaking effect did not last as long as the reduction in impurity influx after carbonization.

As shown in Fig. 3, recycling inferred from $D_{\alpha}$ emission, $\bar{n}_{e}$, and $d \bar{n}_{e} /$ ut were lower while the external gas fueling increased for discharges in the first session after carbonization with peaked H-mode density profiles when compared to discharges in a subsequent session which exhibited less density peaking.

Since the energy confinement time, $\tau_{\mathrm{E}}$, is similar, it is unlikely that changes in $\tau_{\mathrm{p}}$ can totally account for the reduction in $\mathrm{D}_{\alpha}$ emission of nearly a factor of 10 . During beam heating, the external fueling terms, $S_{\text {gas }}$ and $S_{\text {beam }}$ are the same before and after carbonization so lower density must be due to a decrease in wall fueling. A reduction in the recycling coefficient, $R$, may account for the observed decrease in $\overline{\mathrm{n}}_{e}$ and $\mathrm{d} \bar{n}_{e} / \mathrm{dt}$. However a better model than the simple global particle confinement is needed to describe these changes in recycling. 
It is somewhat surprising that recycling would be reduced after carbcinization since a deuterium rich carbon film is deposited during the methane glow. Nurnerous experiments have shown that carbonization can produce a deuterium or hydrogen rich carbon film with $\mathrm{D} / \mathrm{C} \sim 0.4[5,12,13]$. This could lead to excessive fueling of the discharge, $R>1$, as observed in some other carbonized tokamaks $[14,15,16]$. However, baking and HeGWC can desorb deuterium from the surface of these films lowering the recycling coefficient so that the wall acts as a pump. This has been observed in both carbon films and graphite $[2,3,5,17,18]$ and may account for the low recycling initially observed in DIII-D after the first carbonization.

The number of discharges for which carbonization reduces impurity influx depends upon many parameters, e.g. plasma current, discharge shape and neutral beam power. As discussed in Section 3d, after the second carbonization, oxygen returns to $86 \%$ of its pre-carbonization level after 130 discharges during high current operation while the increase in nickel is considerably less. Nickel impurity influx remained below $\sim 30 \%$ of the pre-carbonization level until $\sim 70$ discharges after the second carbonization during a period of high current operation.

Carbonization of the DIII-D tokamak has demonstrated the importance of plasma wall interactions in controlling core plasma characteristics. After carbonization the parameter region in which DIII-D can operate has been enhanced with the addition of high current divertor operation up to $3 \mathrm{MA}$, high beta full field operation, enhanced hot ion $\mathrm{H}$-mode [10], peaked density profiles during $\mathrm{H}$-mode discharges and higher power IBW injection. It is important to note that the maximum plasma current and stored energy which were achieved during this campaign were not limited by plasma wall interactions but rather by limitations on the power supplies for the field shaping coils. No evidence of carbon blooms [19] were observed. Although additional experiments are needed, it appears that the peaked density $\mathrm{H}$-mode profiles which have been observed after carbonization are produced by a reduction in impurities, primarily oxygen anci nickel, accompanied by a reduction in the recycling coefficient, 
R. A baffled divertor with active pumping in the baffle region can reduce the effective particle fueling without the disadvantage that the fueling rate changes nver a period of time. The DIII-D advanced divertor project plans to incorporate such a pumped divertor [20] and with this pumping, peaked density pro fles may be also be achieved.

\section{Acknowledgements}

We wish to acknowledge the support of the DIII-D operations DIII-D neutral beam, and DIII-D physics groups in accomplishing this work. Wc also appreciate the support of Euratom by allowing the collaboration of Prof. J. Winter during this experiment. This work was supported under DOE contract DE-AC03-89ER51114. 


\section{References}

[1] J. Winter, J. Nucl. Mater. 145-147 (1987) 131.

[2] H.F. Dylla, et al., Nucl. Fusion 27, (1987) 1221.

[3] G.L. Jackson, T.S. Taylor, and P.L. Taylor, General Atomics Report GA-A19891 (1.990) and submitted to Nucl. Fusion.

[4] J. Winter, J. Nucl. Mater. 161 (1989) 265.

[5] J. Winter, F. Waelbroeck, P. Wienhold, et al., Surface Conditioning of Vacuum Systems, AIP Conference Proceedings No. 199 (America.1 Institute of Physics, New York, 1990) 50.

[6] G.L. Jackson, T.S. Taylor, P.L. Taylor, and P.I. Petersen, ibid, 39.

[7] P. Wienhold and J. Littmark, Proceedings E-MRS Symposium Amorphous Hydrogenated Carbon Films, Strasbourg, 1987 XVII, (Les Editions de Physique, Paris, 1987) 44.

[8] L.L. Lao, H. St.John, R.D. Stambaugh, et al., Nucl. Fusion 25 (1985) 1611.

[9] D.P. Schissel, J.C. DeBoo, R.J. Groebner, et al., in the proceedings of the seventeenth European Physical Society Conference - $n$ Controlled Fusion and Plasma Heating, Amsterdam, June 25-27, 1990.

[10] K.H. Burrell, R.J. Groebner, T.N. Carlstrom, et al, in the proceedings of the seventeenth European Physical Society Conference on Controlled Fusion and Plasma Heating, Amsterdam, June 25-27, 1990.

[11] J.L. Luxon, et al., ibid.

[12] F. Waelbroeck, T. Banno, H.G. Esser, et al., J. Nucl. Mater. 162-164, (1989) 496. 
[13] B.M.U. Scherzer, M. Wielunski, W. Moller, et al., Nucl. Instr. and Meth. in Phys. Res. B33, (1988) 714.

[14] G. Fussmann, J. Nucl. Mater. 145-147 (1987) 96.

[15] N. Noda, Y. Hori, K. Masai, et al., J. Nucl. Mater. 162-164, (1989) 709.

[16] V.M. Chicherov, Yu.V. Esipchuk, S.A. Grashin, et al., ibid, (1989) 737.

[17] Y. Hirooka, W.K. Leung, R.W. Conn, et al., J. Vac. Sci. Technol. A6, (1988) 2965.

[18] W.R. Wampler and S.M. Mayers, J. Nucl. Mater. 111-112, (1982) 616.

[19] H.F. Dylla, et al., these proceedings.

[20] M.A. Mahdavi, these proceedings. 

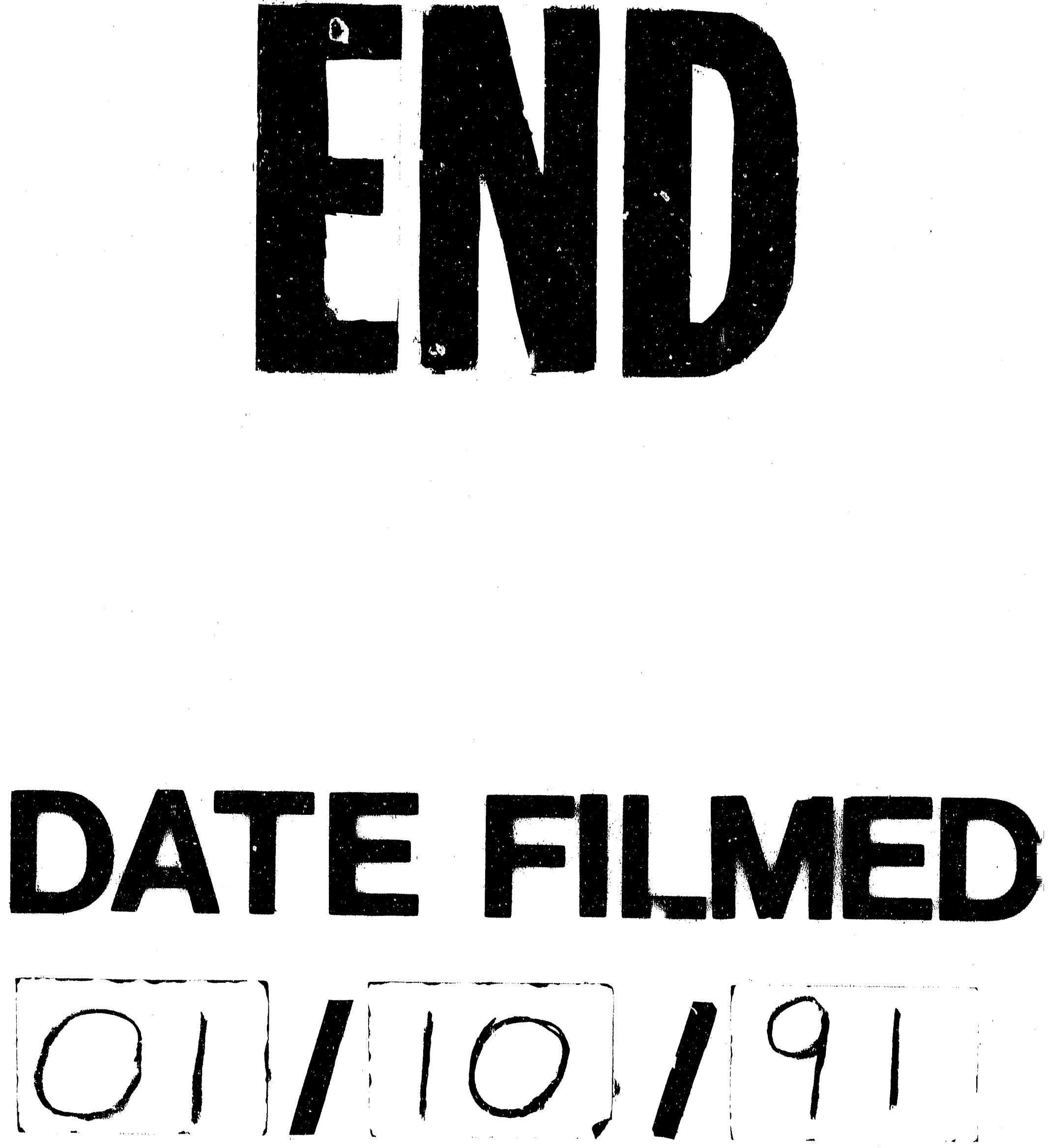


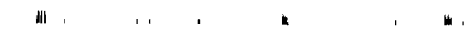

